\title{
Comparison of STIS and SNAP Spectrograph Throughputs
}

\author{
Greg Aldering \\ E.O. Lawrence Berkeley National Lab \\ June 3, 2002; updated June 28, 2002
}

This is a comparison of the measured throughput of STIS on HST versus what we might expect from the spectrograph on SNAP. The principle reference is Woodgate et al. (1998) PASP, 110, 1183. Additional material was taken from the STIS Handbook, available on-line at www.stsci.edu. The goal is to demonstrate that there are sound reasons to expect better performance for a SNAP spectrograph (even one with a grating) than would be expected by scaling from HST+STIS.

In Table $1 \mathrm{I}$ attempt to reverse-engineer the STIS system throughput at $0.77 \mu \mathrm{m}$ by using published or assumed efficiencies for constituent STIS optical components. In particular, the CCD efficiency is given Table 10 of Woodgate et al and the Al efficiency is the textbook value for fresh Al. The grating efficiency, and the dewar window throughput are assumed quantities. The behavior with wavelength of several STIS components is shown in Figure 1. The HST OTA throughput is not included in Table 1. The reference wavelength of $0.77 \mu \mathrm{m}$ was chosen since Woodgate et al quote their efficiency at this wavelength.

Table 1: STIS Efficiency @ $0.77 \mu \mathrm{m}$

\begin{tabular}{|l|c|c|l|}
\hline Component & $\begin{array}{c}\text { Component } \\
\text { efficiency }\end{array}$ & $\begin{array}{c}\text { Cumulative } \\
\text { efficiency }\end{array}$ & Notes \\
\hline 4 Al reflections & 0.55 & 0.55 & aberration corr+coll+cam each $86 \%$ reflectivity \\
grating & 0.65 & 0.36 & at peak, including $86 \%$ reflectivity \\
dewar window & 0.98 & 0.35 & \\
CCD & 0.51 & 0.18 & from Table 10 of Woodgate et al. \\
\hline
\end{tabular}

The measured throughput for the G750L grating at $0.77 \mu \mathrm{m}$ given in Table 1 of Woodgate et al 1998 is $10.0 \%$, while the G750M gratings has a measured throughput of $14.8 \%$. (The lower efficiency of G750L compared to G750M is due to a Lyot stop) The value of $14.8 \%$ is in good agreement with the ab initio estimate of $18 \%$ in Table 1. Indeed, the STIS efficiency in the G750M grating at $0.77 \mu \mathrm{m}$ given by the HST calibration pipeline tables (i.e. in SYNPHOT) is $17.6 \%$ slightly higher than the value published in Woodgate et al. and in excellent agreement with the $a b$ initio estimate. This suggests that the assumed efficiencies of the spectrograph components are plausible.

In Table 2 I show how a similar spectrograph on SNAP would fare relative to STIS at $0.77 \mu \mathrm{m}$. Here the OTA efficiencies for both telescopes are included. Basically, we should expect SNAP+spectrograph to be roughly $4 \times$ more efficient than HST + STIS. 


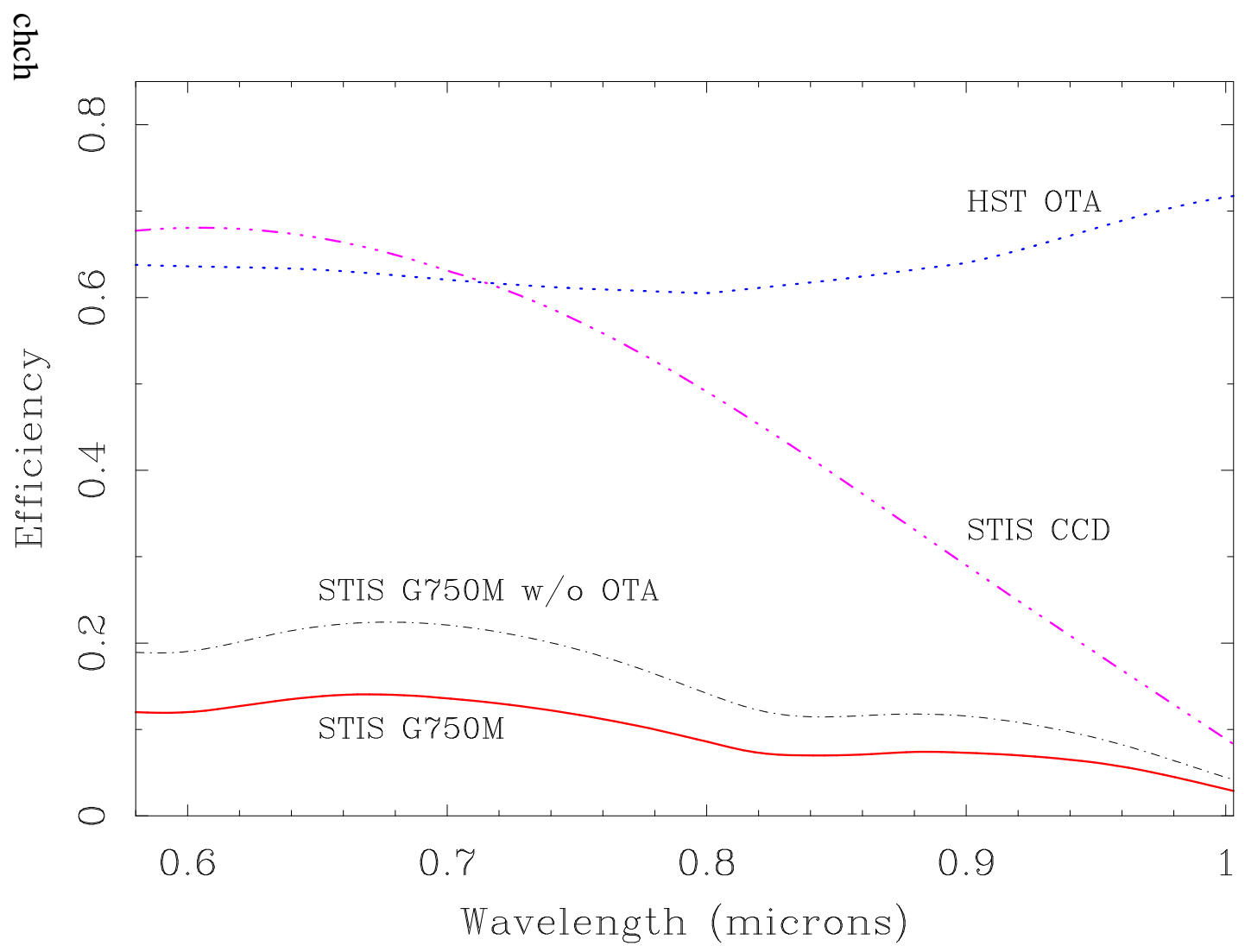

Fig. 1.- Efficiency of key STIS components, plus the total system throughput including the optical telescope assembly.

SNAP's spectroscopy advantage arises from better efficiency for each of the key components: OTA, spectrograph optics, disperser efficiency, and detector efficiency. These efficiency advantages come about in a traceable fashion and for many components derive from the specific trades allowed by SNAP's dedicated SN program relative to a generic observing program.

One interesting point to note is the lower OTA throughput for HST. This is not strongly dependent on wavelength. The HST mirror is $2.4 \mathrm{~m}$ with a $0.6 \mathrm{~m}$ diameter hole, but a $0.79 \mathrm{~m}$ diameter shadow from the secondary baffle. This gives a geometrical throughput of $89 \%$. This is actually better than for SNAP, where the aperture is only $84 \%$ unobscured due to the large field of view. Two reflections off $\mathrm{Al}$ at $86 \%$ each should then give a total throughput of $66 \%$ for HST, in fair agreement with the published value of $61 \%$. Despite the greater number of mirrors in the SNAP OTA, the efficiency is higher than for HST because the losses from Ag are so much smaller than for $\mathrm{Al}$. 
Table 2: STIS vs. SNAP-like Spectrograph @ $0.77 \mu \mathrm{m}$

\begin{tabular}{|l|c|c|l|}
\hline Component & STIS & SNAP & \\
\hline OTA & 0.61 & 0.77 & 2 Al reflections vs 4 Ag reflections + obs \\
spectro. optics & 0.55 & 0.83 & 4 Al reflections vs 9 Ag reflections \\
grating/prism & 0.65 & 0.81 & grating on blaze vs prism for SNAP \\
instrument window & 0.98 & 0.98 & \\
CCD & 0.51 & 0.92 & \\
\hline & 0.11 & 0.47 & \\
\hline
\end{tabular}

\section{Acknowledgements}

I would like to thank Bruce Woodgate (GSFC), the STIS principle investigator, along with Chuck Bowers (GSFC) for useful discussions. This work is supported by the U.S. Department of Energy. 